NASA/TM-2003-212112

AIAA-2003-1303

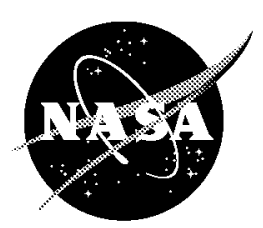

\title{
The Light Microscopy Module Design and Performance Demonstrations
}

Susan M. Motil, John H. Snead, and DeVon W. Griffin

Glenn Research Center, Cleveland, Ohio

Edward A. Hovenac

Northrop Grumman Information Technology, Brook Park, Ohio 
The NASA STI Program Office ... in Profile

Since its founding, NASA has been dedicated to the advancement of aeronautics and space science. The NASA Scientific and Technical Information (STI) Program Office plays a key part in helping NASA maintain this important role.

The NASA STI Program Office is operated by Langley Research Center, the Lead Center for NASA's scientific and technical information. The NASA STI Program Office provides access to the NASA STI Database, the largest collection of aeronautical and space science STI in the world. The Program Office is also NASA's institutional mechanism for disseminating the results of its research and development activities. These results are published by NASA in the NASA STI Report Series, which includes the following report types:

- TECHNICAL PUBLICATION. Reports of completed research or a major significant phase of research that present the results of NASA programs and include extensive data or theoretical analysis. Includes compilations of significant scientific and technical data and information deemed to be of continuing reference value. NASA's counterpart of peerreviewed formal professional papers but has less stringent limitations on manuscript length and extent of graphic presentations.

- TECHNICAL MEMORANDUM. Scientific and technical findings that are preliminary or of specialized interest, e.g., quick release reports, working papers, and bibliographies that contain minimal annotation. Does not contain extensive analysis.

- CONTRACTOR REPORT. Scientific and technical findings by NASA-sponsored contractors and grantees.
- CONFERENCE PUBLICATION. Collected papers from scientific and technical conferences, symposia, seminars, or other meetings sponsored or cosponsored by NASA.

- SPECIAL PUBLICATION. Scientific, technical, or historical information from NASA programs, projects, and missions, often concerned with subjects having substantial public interest.

- TECHNICAL TRANSLATION. Englishlanguage translations of foreign scientific and technical material pertinent to NASA's mission.

Specialized services that complement the STI Program Office's diverse offerings include creating custom thesauri, building customized databases, organizing and publishing research results... even providing videos.

For more information about the NASA STI Program Office, see the following:

- Access the NASA STI Program Home Page at http://www.sti.nasa.gov

- E-mail your question via the Internet to help@sti.nasa.gov

- Fax your question to the NASA Access Help Desk at 301-621-0134

- Telephone the NASA Access Help Desk at 301-621-0390

- Write to: NASA Access Help Desk NASA Center for AeroSpace Information 7121 Standard Drive Hanover, MD 21076 
NASA/TM-2003-212112

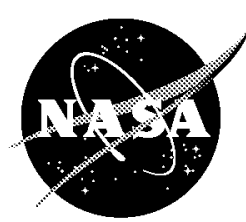

\section{The Light Microscopy Module Design and Performance Demonstrations}

Susan M. Motil, John H. Snead, and DeVon W. Griffin

Glenn Research Center, Cleveland, Ohio

Edward A. Hovenac

Northrop Grumman Information Technology, Brook Park, Ohio

Prepared for the

41st Aerospace Sciences Meeting and Exhibit

sponsored by the American Institute of Aeronautics and Astronautics

Reno, Nevada, January 6-9, 2003

National Aeronautics and

Space Administration

Glenn Research Center

January 2003 


\section{Acknowledgments}

The Microgravity Science Division of the NASA Glenn Research Center is developing both the FIR and LMM. The authors would like to acknowledge the work and dedication of the Northrop Grumman development team. Through their dedication and innovativeness, the FIR and LMM will be unique experiment facilities for carrying out fluids physics experiments on board the International Space Station.

This report is a formal draft or working paper, intended to solicit comments and ideas from a technical peer group.

This report contains preliminary
findings, subject to revision as
analysis proceeds.

Available from

NASA Center for Aerospace Information 7121 Standard Drive

Hanover, MD 21076
National Technical Information Service 5285 Port Royal Road Springfield, VA 22100

Available electronically at htto://gltrs.grcnasa.gov 


\title{
THE LIGHT MICROSCOPY MODULE DESIGN AND PERFORMANCE DEMONSTRATIONS
}

\author{
Susan M. Motil, John H. Snead, and DeVon W. Griffin \\ National Aeronautics and Space Administration \\ Glenn Research Center \\ Cleveland, Ohio 44135 \\ Edward A. Hovenac \\ Northrop Grumman Information Technology \\ Brook Park, Ohio 44142
}

\begin{abstract}
$\underline{\text { Abstract }}$
The Light Microscopy Module (LMM) is a state-of-theart space station payload to provide investigations in the fields of fluids, condensed matter physics, and biological sciences. The LMM hardware will reside inside the Fluids Integrated Rack (FIR), a multi-user facility class payload that will provide fundamental services for the LMM and future payloads. LMM and FIR will be launched in 2005 and both will reside in the Destiny module of the International Space Station (ISS).
\end{abstract}

There are five experiments to be performed within the LMM. This paper will provide a description of the initial five experiments; the supporting FIR subsystems; LMM design, capabilities and key features; and a summary of performance demonstrations.

\section{$\underline{\text { Introduction }}$}

The Light Microscopy Module (LMM) will be the first integrated payload into the Fluids Integrated Rack (FIR), and will conduct five fluid physics experiments onboard the International Space Station (ISS). The FIR is one of two racks that make up the Fluids and Combustion Facility (FCF), ${ }^{1}$ and is scheduled for launch in July of 2005 aboard STS flight UF-5. The LMM is planned as a remotely controllable on-orbit subrack facility, allowing flexible scheduling and operation of fluids and potential biotechnology experiments. Integrated with the FIR, as shown in Figure 1, the LMM will receive power, communications, air and water cooling, vacuum exhaust, avionics, image processing, data storage, and additional science diagnostic hardware provided by the FIR rack. The LMM will be installed on-orbit, and remain in the FIR for a period of two years performing five separate fluid physics experiments.

The LMM is a sophisticated, modified commercial research imaging light microscope with laser diagnostics hardware and interfaces to conduct on-orbit colloid, fluid physics, and potential biological experiments, creating a one-of-a-kind, state-of-the-art research facility. The LMM will provide imaging techniques of color video microscopy, bright field, dark field, phase contrast, differential interference contrast (DIC), interferometry, spectrophotometry, and confocal microscopy combined in a single configuration. Singlebeam scanning laser tweezers are integrated with the diagnostics to perform sample particle manipulation, patterning, and localized heating.

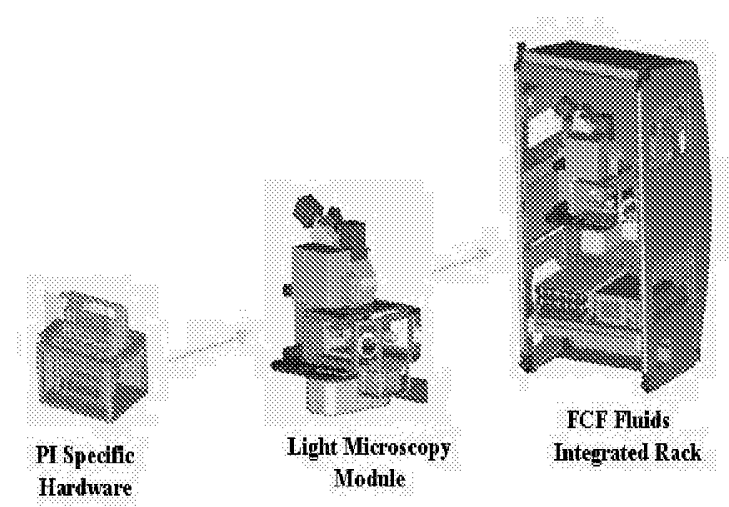

Figure 1 - Integrated LMM and FIR Design

$\underline{\text { Initial Experiments }}$

The LMM will be making full use of the flexibility of the FIR, its diagnostic hardware, and numerous interfaces. The multi-user, mini-facility, integrated concept supports specific research and scientific investigations by the Microgravity Research Program as part of the Office of Biological and Physical Research (OBPR). Within the FIR, an initial complement of five fluid physics investigations will utilize the LMM instrument. These experiments are the "Constrained Vapor Bubble" (Peter C. Wayner, Rensselaer Polytechnic Institute), "Physics of Hard Spheres Experiment-2" (Paul M. Chaikin, Princeton University), "Physics of Colloids in Space-2" (David A. Weitz, Harvard University), "Low Volume Fraction Entropically Driven Colloidal Assembly" (Arjun G. Yodh, the University of Pennsylvania), and "Micromechanics of Magnetorheological Fluids" 
(Alice P. Gast, Massachusetts Institute of Technology). While meeting the needs of those investigations, the LMM team is attempting to provide sufficient design flexibility to facilitate supporting additional researchers.

The primary mission of the LMM is to accommodate these initial five experiments, and meet the scientific objectives defined by the Principal Investigators (PIs). In addition, the LMM utilizes FIR common hardware, architecture (including interface design) and subsystems to minimize cost and increase redundancy. The LMM design permits on-orbit reconfiguration and maintenance to support follow-on fluid physics or biotechnology experiments, while minimizing up-anddown-mass. In order to support five PIs within a twoyear on-orbit time, the LMM will be designed for autonomous operation to minimize crew time needed to set-up, perform and acquire scientific data from experiments. Finally, the LMM is designed for long life installation on FIR. Key components are selected as long life items, on-orbit replaceable modules to enable upgrades, incorporate new technology and/or provide for on-orbit reliability for the two-year design life of the LMM.

\section{Constrained Vapor Bubble}

The Constrained Vapor Bubble (CVB) investigation will be the first experiment performed on-orbit within the LMM. The objective of this experiment is to better understand the physics of evaporation and condensation as they affect the heat transfer processes in a constrained vapor bubble heat exchanger. The CVB heat exchanger consists of a quartz cuvette, enclosed and partially filled with a liquid. When a temperature difference is applied to the ends of the cuvette, evaporation occurs at the hot end and condensation at the cold end, resulting in a very effective heat transfer device. $^{2,3}$

The PI seeks to determine the stability, fluid flow characteristics, the average heat transfer coefficient in the evaporator, and the heat conductance of the CVB as a function of the heat flow rate and vapor volume. The immediate goal is to detail the characteristics of the transport processes in the curved liquid film. The local conditions under which cavitation and instability occur with the formation of a dry region will be determined as a function of heat flux, film thickness, and stress. Both completely wetting (Pentane) and partially wetting (Ethanol) fluids will be studied and compared. The LMM provides interferometry to observe the expected interfacial phenomena.

\section{Physics of Hard Spheres Experiment -2}

The experiment objective for the Physics of Hard Spheres Experiment - 2 (PHASE-2) is to further investigate the nucleation, growth, structure, dynamics, and rheology of colloidal crystals. ${ }^{4}$ The PIs seek to observe the effect of hard sphere parametric conditions on the equilibrium phase diagram, and how colloidal systems respond to applied fields. ${ }^{5,6}$ PHASE-2 consists of microscopic and mesoscopic manipulation and control of colloidal samples to probe the essential features of both the initial hard sphere disorder-order transition and the final ordered solid phase. Samples of various volume fractions will permit measurements from the disordered fluid phase, through the coexistence region, into the fully crystalline solid, and beyond into a glass-like phase. Achieving these measurements will require application of external fields, including temperature gradients and electrical fields, and force application via the optical tweezers. The dispersions will consist of polymethylmethacrylate colloidal hard spheres, in the 0.65 to $1.0 \mu \mathrm{m}$ diameter range, in an index-matched fluid.

Microscopy will allow for the observation of the position of individual colloidal particles and thus the determination of local crystalline or liquid structure, defect structure, observation of nucleation and growth of individual crystallites, the spontaneous formation of dendrites, and other imposed patterns, induced local melting and freezing, and dynamics of liquid-crystal, liquid-glass and solid-glass interfaces. The applied fields will allow for controlled nucleation and growth, induced metastable phases, susceptibility to electric fields, and rheology measurements.

The large number of samples to be tested has lead the LMM to develop a platen containing up to 120 sample cells, as shown in Figure 2. About $1 \mu \mathrm{l}$ of sample fluid is contained in each sample cell (Figure 3). This design allows the contents of many cells to be examined under a variety of conditions before a crew member is needed to exchange platens.

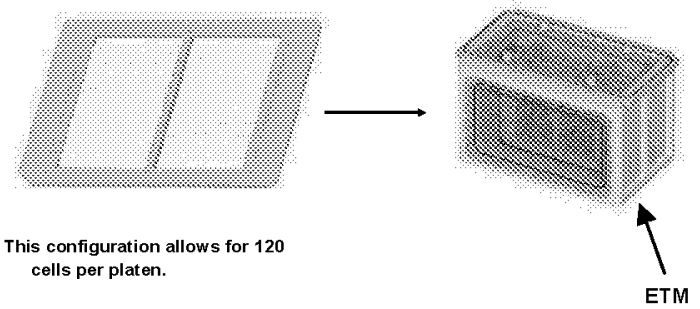

Figure 2 - LMM sample platen 


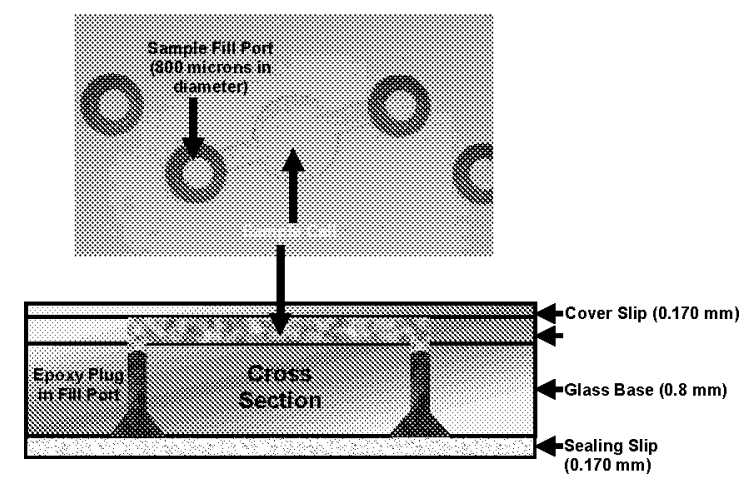

Figure 3 - LMM sample wells

$\underline{\text { Physics of Colloids in Space }-2}$

The goals of Physics of Colloids in Space - 2 (PCS-2) are twofold: to carry out further investigation of critical, fundamental problems in colloid science, and to further develop the evolving field of "colloid engineering". Colloid Engineering is defined as creating materials with novel properties using colloidal particles as the precursors. This experiment continues the research from Physics of Colloids in Space (PCS), which has operated on the International Space Station. ${ }^{7}$

PCS-2 will focus on two classes of colloidal samples. The first is binary alloys. These contain a mixture of two different sized acrylic spheres, ranging from $10 \mathrm{~nm}$ to $5 \mu \mathrm{m}$, dispersed in a liquid. The choice of size ratio, concentration ratio, and total particle volume fraction will be based on the results from precursor colloidal flight experiments. The second set of samples will be a mixture of colloidal particles with polymers. The addition of the polymers induces a controllable attractive force due to depletion between the colloidal particles. This attractive force facilitates the formation of other structures. In addition, the presence of an attractive force induces new phase behavior in the colloidal suspensions.

The LMM optical microscope will allow the properties of the materials to be measured, and allow a degree of proactive control over the structures and properties of the materials produced. The microscope will be equipped to allow direct visualization of the particles in real space, using bright field and differential interference contrast (DIC) imaging, as well as fluorescent imaging. The microscope will also be equipped with laser tweezers to manipulate the structures. In addition, strongly scattering samples will be studied and $3 \mathrm{D}$ real-space information provided via confocal microscopy. Because of the very small sample volumes required, the LMM will be able to examine up to 120 samples, allowing the requisite sample variation to be achieved. Sample cells are identical to the PHASE-2 experiment, and shown in Figures 2 and 3.

\section{Low Volume Fraction Entropically Driven Colloidal Assembly Experiment}

The Low Volume Fraction Entropically Driven Colloidal Assembly (LФCA) experiment aims to create new colloidal crystalline materials, study the assembly of these materials, measure their optical properties, and then solidify the resulting structures so that they can be brought back and studied on earth. ${ }^{8}$ The experiment consists of sample preparation, microscopic observations of crystal growth, photonic characterization of samples, and sample solidification. The latter two parts will only be carried out on selected samples. Samples will consist of colloidal particles with diameters ranging from 0.4 to 3.0 microns suspended in water or various organic fluids. The particles will be polymer-based spheres such as PMMA or polystyrene (PS), silica with or without embedded fluorophores, semiconductors, or novel polymer-based spheres that have hollow interiors or interiors filled with magnetic or metal grains. The PI's interest in investigating colloidal crystal growth and formation includes the creation of novel structures and photonic materials (e.g., ultralow-noise light sources, switches, and strong ceramics) of industrial importance.

The samples are contained in the same configuration as shown in Figures 2 and 3. A grating template will be bonded to the coverslip, which biases the growth process so that crystals with random stacking are not formed. The sample volumes will be about 1 to 5 microliters. At the beginning of the experiment, the samples will be homogenized and the evolution of crystal structures will be observed using the LMM microscope. Crystal growth processes will be monitored and images acquired and stored. This will enable the determination of crystal structure and crystal quality. Those observations will be correlated with sample volume fractions. In aqueous suspensions, the solidification process is facilitated by UV light-induced polymer gelation. In organic solvents, a similar procedure will be developed. Generally, the materials that will be synthesized cannot be made on earth because of sedimentation. Finally, it is anticipated that the solidified structures will survive re-entry to earth's gravitational field, and their optical, magnetic, and electrical properties can be further studied in detail upon return.

\section{$\underline{\text { Micromechanics of Magnetorheological Fluids }}$}

The Micromechanics of Magnetorheological Fluids ( $\mu$ MRF) microgravity experiment will study the 
rheological properties and long range lateral attraction in Magnetorheological (MR) fluids. It will also investigate the rheological properties of composite chains formed through depletion-induced coalescence. The study involves bending and stretching deformations of dipolar chains and columns in concentrated suspensions to provide qualitative and quantitative measurements of the microstructural mechanics. Microgravity conditions offered in the ISS eliminate the need for density matched MR suspensions and allows for higher concentrations of samples, which broadens the range of aggregate sizes to be investigated. ${ }^{9}$

Single trap experiments have been designed to probe the micromechanics of chains attached to a magnetic wall. These experiments lend themselves to using the optical tweezers provided by the LMM. The laser tweezer subsystem is used to directly stretch the chains and provide quantitative force measurements, contributing to the study of MR microstructures. This particle manipulation will be observed through traditional video microscopy techniques.

\section{Supporting Fir Subsystems}

The LMM will utilize six major FIR subsystems to accommodate the suite of fluids physics experiments. These subsystems are: structural, environmental, electrical, gaseous, command and data management and diagnostics. The FIR provides LMM with a stable mounting platform, and additionally allows for acoustic emission attenuation, minimal air/thermal exchange with the cabin, reduced airborne contaminate migration into the rack, and prevents stray light from entering or leaving the rack. ${ }^{10}$

\section{$\underline{\text { Structural Subsystem }}$}

The centerpiece of the FIR structural subsystem is the optics bench. The optics bench provides a mounting surface for FIR light sources and avionics packages on the back of the bench and for LMM hardware on the front of the bench. LMM will be installed directly to the optics bench utilizing threaded mounting holes. The optics bench is designed so it can be translated out of the rack and rotated forward, as shown in Figure 4, to allow the crew easy access to the LMM and FIRprovided hardware. During powered operations the LMM is in the stowed position and the rack doors are closed. In the un-powered mode, the rack doors can be opened and the optics bench translated forward. Fully translated out, the front surface of the optics bench will extend past the front of the rack slightly into the aisle way of the cabin providing easy access to the front of the bench. The bench can be rotated forwarded ninety degrees providing access to the back of the bench for procedures such as replacing FIR hardware or routing a fiber optic cable from the light sources on the back of the bench to LMM hardware on the front of the bench.

The Active Rack Isolation System (ARIS) enhances the micro-gravity environment for LMM experiments within the FIR. ARIS minimizes vibratory transmission to and from the rack. ARIS utilizes three accelerometers to measure the micro-gravity environment in the rack. Based on the measured accelerations ARIS applies reactive forces, between the FIR and ISS, through eight actuators to minimize transmission of vibratory disturbances. ARIS will be particularly important for the first LMM experiment, CVB, which requires micro-gravity levels below $10^{-4} \mathrm{~g}$ (dc level).

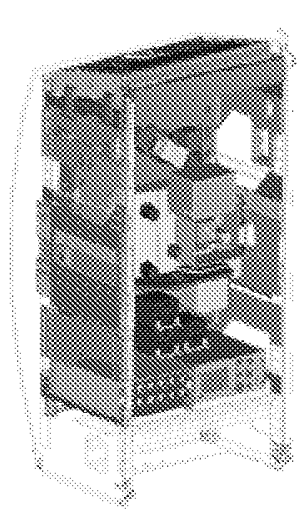

Stowed

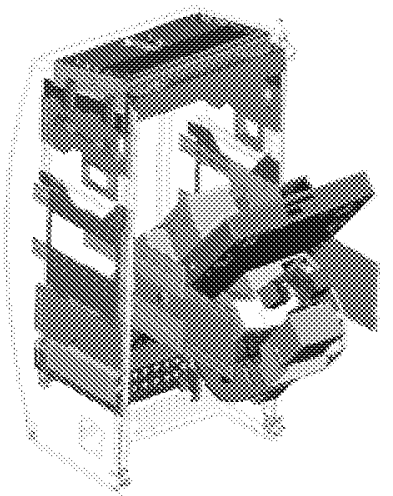

Fully Translated and Partially

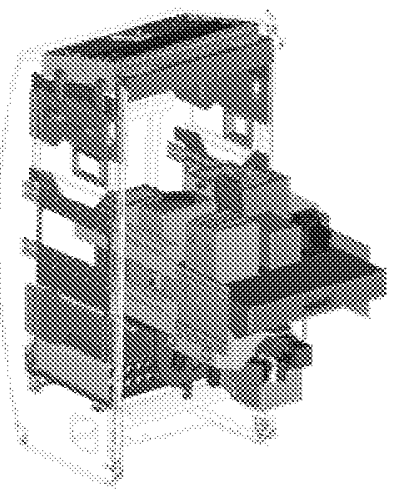

Fully Translated and Fully Rotated

Figure 4 - FIR Optics Bench Deployment with LMM Mounted to the Bench 


\section{Environmental Subsystem}

LMM requires a stable temperature environment that will allow the optics to properly function and aid LMM in maintaining PI samples at a constant temperature. The FIR-provided environmental subsystem will utilize air and water to remove heat generated by the FIR and LMM. The air thermal control system will provide a temperature controlled environment for the payload. The control set-point, which can be located anywhere within the payload volume, is selectable between $20{ }^{\circ} \mathrm{C}$ and $30^{\circ} \mathrm{C}$ by the payload. Air will be circulated throughout the rack and will be cooled by the Air Thermal Control Unit (ATCU), an air-water heat exchanger located in the top of the rack. The design point for the air-cooling system is $1650 \mathrm{~W}$ at 220 cubic feet per minute, however, the system can operate at other heat loads and air flow rates. Cool air will leave the heat exchanger and flows over the front of the optics bench cooling LMM hardware. After cooling the hardware on the front of the bench, the air will cool the hardware located below and on the back of the optics bench. The warm air then returns to the ATCU through the optics bench.

LMM will also utilize cooling water for additional conditioning of the air around its samples and for removing heat from sample cooler and heaters. Cooling water from the ISS moderate temperature loop enters the rack and is split into two cooling loops. One loop is dedicated to the FIR hardware the other loop is for payload hardware. Each loop is capable of removing $3 \mathrm{~kW}$ of heat. The water inlet temperature to the rack is nominally $16.6^{\circ} \mathrm{C}$. The ISS regulates the water mass flow rate through the rack in order to maintain nominally a $20^{\circ} \mathrm{C}$ delta between the water inlet and outlet temperatures.

\section{$\underline{\text { Gas Interface Subsystem }}$}

LMM will use the Vacuum Exhaust System (VES) to verify seals on-orbit. An ISS crew member will hook the VES up to the LMM, pull a slight vacuum, and measure the decay over time to verify integrity of the seal. FIR provides payloads access to the ISS vacuum system, through the Gas Interface Panel (GIP). The VES has a high throughput and is intended for pulling a chamber or system from pressures up to $276 \mathrm{kPa}$ down to a vacuum $(0.13 \mathrm{~Pa})$.

\section{$\underline{\text { Electrical Subsystem }}$}

LMM receives 28 VDC power through the FIR. The Electrical Power Control Unit (EPCU) is the heart of the FIR electrical subsystem. All power from ISS will flow through the EPCU. The EPCU will provide power management and control functions, as well as fault protection. The EPCU will take $120 \mathrm{VDC}$ from ISS power bus to provide 48 channels of 28 VDC 4 A fault protected circuits to the FIR and LMM and six 120 VDC $4 \mathrm{~A}$, not being used by LMM.

\section{$\underline{\text { Command and Data Management Subsystem }}$}

The FIR Command and Data Management subsystem (CDMS) provides command and data handling for both the facility and LMM. The main components of the FIR CDMS are the Input Output Processor (IOP), the Image Processing and Storage Unit (IPSU), the Fluids Science Avionics Package (FSAP) and the Mass Data Storage Unit (MDSU).

The Input Output Processor (IOP) will provide the link from the FIR to the ISS command and data management system via the MIL-STD-1553B, Ethernet, analog video and the High Rate Data Link interfaces. The IOP provides the overall command and data management functions for the FIR. The IOP controls the core facility subsystems such as the ARIS, EPCU and the environmental control subsystem. The IOP will receive and store science data from the FSAP, image data from the IPSUs and ancillary rack data. The IOP will store data on two removable $182 \mathrm{~GB}$ hard drives. This data can be downlinked or the hard drives can be removed and replaced with new ones. The IOP can route analog video directly to the ISS for downlinking or storage on an ISS video recorder.

The FIR will be able to accommodate two Image Processing and Storage Units (IPSUs) with slightly different features. Both IPSUs will perform diagnostic control and image processing and storage functions. The Fluids Science Avionics Package controls each IPSU. Both IPSUs will be able to provide control for a camera, receive image data from the camera, and process or compress image data as necessary. LMM uses existing FIR software and generates custom software to process and compress image data. One IPSU can receive images from a digital camera through a custom Serial Data Link (SDL) interface or through a standard IEEE-1394 interface. The other IPSU will have an analog frame grabber in place of the serial data link interface. The serial data link converts image data transmitted from a digital camera through a fiber back to a digital signal so it can be processed by the IPSU. LMM cameras have an IEEE-1394 interface and are controlled through this interface. Additionally, LMM utilizes a FIR-provided Toshiba Camera for general microscopy operations. This camera utilizes the analog frame grabber interface and is controlled by the Control Area Network (CAN) bus provided through the Generic Package Interface (GPI) connectors. The IPSU will be 
capable of receiving raw image data at $64 \mathrm{MB} / \mathrm{s}$. At this rate the IPSU will be capable of receiving a 1024 pixel by 1024 pixel, 16 bit image at 30 frames per second. The IPSU will be capable of performing automated real-time image analysis in order to support real-time activities such as object tracking. The IPSU has two $18 \mathrm{~GB}$ hard drives for image storage. Once the data is stored the IPSU will be capable of post processing images. LMM will use the C-IPSU to analysis images and control the microscope optics. In addition LMM processes and compresses images in the $\mathrm{C}$-IPSU reducing the data to a manageable amount.

The Fluids Science and Avionics Package (FSAP) will provide the primary control in carrying out the LMM. Through the FSAP, the LMM will be able to execute an experiment by controlling the FIR diagnostics and avionics packages as well as payload hardware. LMM will download its own custom software into the FSAP for experiment control. The FSAP will be able to command and control the IPSUs via Ethernet. The FASP will provide the following: motion control, analog to digital channels, digital to analog channels, digital inputs and outputs, external and internal triggers, RS-422, analog frame grabber and CAN bus. The FSAP has two $18 \mathrm{~GB}$ hard drives for storing analog and digital data as well as analog video images captured by the analog frame grabber. LMM has access to the FSAP through the PI power/data connectors located on the front of the optics bench.

The Mass Data Storage Unit (MDSU) provides supplemental data storage for the rack. The MDSU will have a data storage capacity of approximately 1 TeraByte. The current design utilizes a six removable hard drives. Within the FIR, the MDSU will be compatible with locations on the front and back of the optics bench. It is an ORU and therefore can be removed from the rack and placed in stowage if not required.

\section{Diagnostics Subsystem}

With the initial deployment of the FIR and through facility upgrades, the FIR will provide a suite of cameras and illumination sources to support a wide range of diagnostic capabilities typically required by fluids physics experiments. As part of the initial deployment, the FIR will provide two illumination sources, $532 \mathrm{~nm} \mathrm{Nd:YAG}$ laser and a white light source for use with the LMM.

LMM will utilize the FIR-provided color camera for imaging samples and will use the images from the camera to control position of its optics. The FIR color camera is a 24 bit, 3-chip Charged Coupled Device
(CCD) analog color camera. The camera has a onethird inch array with a pixel density of 768 pixels $\times 484$ pixels. The camera's frame rate is settable up to 30 frames per second (fps) and shutter speed is settable from 1/60 of a second down to $1 / 10,000$ of a second. In auto shutter mode, shutter speeds down to $1 / 50,000^{\text {th }}$ of a second can be achieved. The color camera will be controlled by the FSAP over the CAN bus. Images from the color camera will be sent to the FSAP via a RS-170A signal for storage. The color camera has a small remote head, $32 \mathrm{~mm}(\mathrm{~W}) \times 40 \mathrm{~mm}(\mathrm{H}) \times 40 \mathrm{~mm}$ (D), to facilitate camera integration in confined areas. The color camera utilizes a standard C-mount for lenses.

LMM will utilize the white light for imaging the samples, spectrophotometry and interferometry. The FIR white light package will be located on the back of the FIR optics bench. The package will be controlled by the FSAP through the CAN bus. The package consists of two $50 \mathrm{~W}$ metal halide bulbs subassemblies. Each subassembly has a fiber optic quick disconnect for attachment of a fiber bundle. Adjusting an aperture knob located on a subassembly will vary the light intensity delivered through the fiber bundle attached to that subassembly. Fibers will be routed to the front of the optics bench from the white light package on the back of the bench through the fiber feed through located in the bench.

Confocal images of crystalline structures require the use of a $532 \mathrm{~nm}$ light source. The FIR $532 \mathrm{~nm} \mathrm{Nd:YAG}$ laser package, located on the back of the FIR optics bench, is connected to the LMM via fiber optic cable. A quick disconnect at the end of the fiber optic cable allows for easy hookup to LMM. The package will be controlled by the FSAP through the CAN bus. The laser is capable of providing a minimum power of $100 \mathrm{~mW}$, with the output power capable of varying from 0 to $100 \%$ of the maximum power. A tap coupler located at the output of laser will measure laser power delivered to the payload

\section{$\underline{\text { LMM Capabilities and Their Performance }}$}

The LMM flight unit features a modified commercial off-the-shelf (COTS) Leica RXA microscope, which is enhanced to operate automatically with some interaction from the ground support staff or the astronaut crew. In addition to video microscopy techniques to record sample features including basic structures and crystal growth dynamics, the microscope is modified and enhanced to provide additional capabilities including: 1) Interferometry, to measure vapor bubble thin film thickness, 2) Laser tweezers for sample particle manipulation and patterning, 
3) Confocal microscopy to provide three-dimensional visualization of sample structures, and 4) Spectrophotometry to measure photonic properties.

The imaging techniques of high resolution color video microscopy, bright field, dark field, phase contrast, differential interference contrast (DIC), fluorescence, spectrophotometry, and confocal microscopy are combined in a single configuration. An experimenter can choose from six (6) objective lenses of different magnifications and numerical aperture to achieve the required science data. This suite of measurements allows a very broad characterization of fluids, colloids, and two-phase media, including biological samples. Sample manipulation technique is integrated with the microscope's single-trap scanning laser tweezers. The LMM utilizes cameras and light sources provided by the FIR to accomplish these imaging techniques.

The LMM provides containment for fluids and shatterable materials. Essential components include an Auxiliary Fluids Container (AFC), which will be attached to the microscope, an Equipment Transfer Module (ETM) that will attach to the AFC, and the microscope itself, as shown in Figure 5. The AFC is an enclosed compartment encompassing the microscope's objective lenses, $\mathrm{x}-\mathrm{y}$ translation and focusing stages, and condenser unit hardware. The AFC is clamped to the microscope and is sealed to provide a clean working space and minimize the risk of contamination to the FCF FIR, ISS, or the experiment samples. The AFC provides large viewing windows; electrical feedthroughs for power, control, and data; gloveports

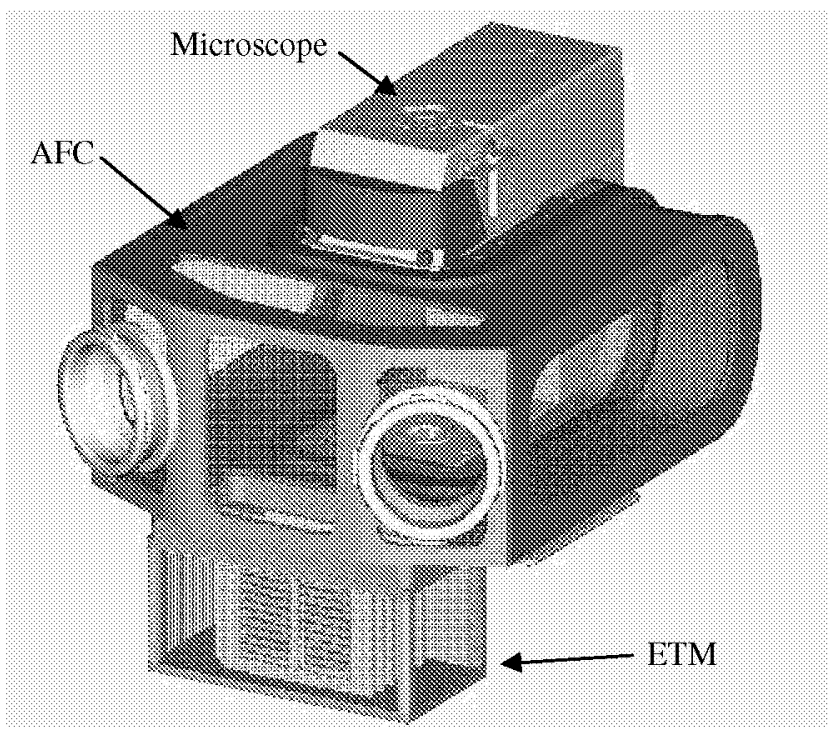

Figure 5 - Integrated microscope, AFC, and ETM for handling and transfer of materials; water cooling lines routed from FIR and an internal fan for air cooling; and a surveillance camera for monitoring experiment operations or sample status.

In general, PI experiment samples will be launched and transported in the ETM, and then the ETM will be attached to the $\mathrm{AFC}$. A crewmember will wear $\mathrm{AFC}$ gloveport gloves, to remove the PI experiments from the ETM, and place the samples on the microscope stage. After the sample has been positioned, remote operation of the microscope $e$ and processing of the samples can begin.

\section{Video Microscopy}

Video microscopy will be performed via transillumination imaging techniques already resident within the Leica RXA microscope itself, namely, bright field, dark field, phase contrast, and differential interference contrast (DIC). A variety of condenser apertures, objectives, and prisms are required to support these techniques, but the Leica RXA comes well suited for the task. Optical microscopy of colloidal suspensions will be performed using the four transillumination techniques.

The microscope will be configured to have a 0.9 Numerical Aperture (NA) condenser and microscope objectives up to $100 \mathrm{x}$ and 1.4 NA (oil immersion) to support the required transillumination techniques. The light source for Köhler illumination is to be provided by the FIR metal halide lamp. Images, such as those shown in Figure 6 , will be captured by the FIR Toshiba color analog camera on the microscope's multi-port imaging head and subsequently downlinked for data processing.

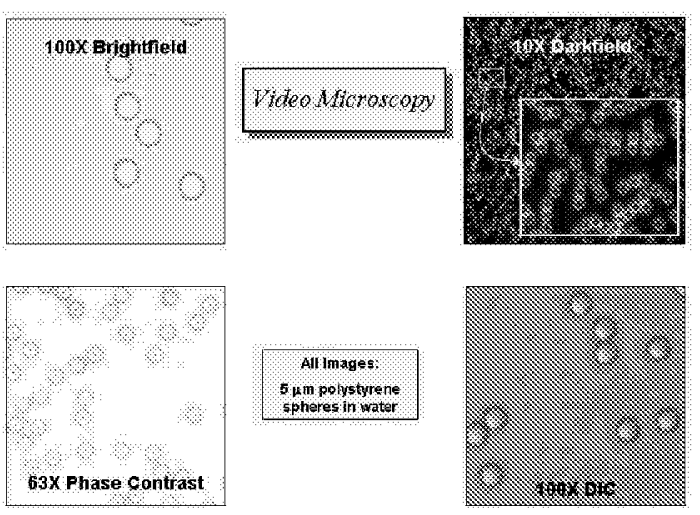

Figure 6-Microscope images of $5 \mu \mathrm{m}$ polystyrene spheres 


\section{$\underline{\text { Interferometry }}$}

Interferometry is used to measure the curvature of the fluid meniscus for the CVB experiment. The light source in epi-illumination provides the required wavelength to create the interference fringes. The light passes through the liquid and partially reflects at all interfaces, including the glass-liquid interface and liquid-vapor interface. The light from the two reflections interferes to form fringes that represent an increase in fluid thickness of half the wavelength of light for each bright and dark pair, as shown in Figure 7. The QImaging Retiga 1300, black and white, 12 bit camera on the multi-port imaging head will capture the images for downlinking and processing. Analyzing the interferometry images will yield a film thickness profile. ${ }^{11}$

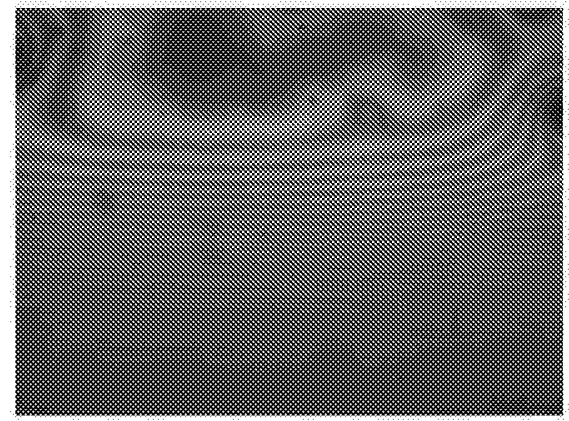

Figure 7 - CVB bubble image at $435 \mathrm{~nm}$

The derivative of the thickness gives the curvature profile, and therefore the pressure field for flow in the liquid film. The thickness will be measured at multiple axial positions along the cuvette. Figure 8 shows the CVB fringe profile as intensity versus pixel number for the image.

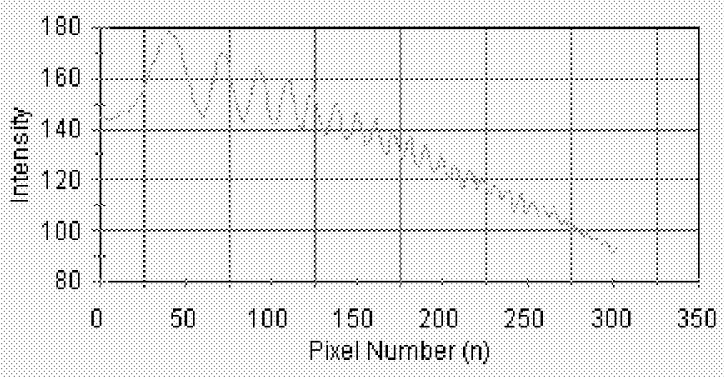

Figure 8 - CVB Fringe Profile

\section{$\underline{\text { Laser Tweezers }}$}

A laser tweezer system will be included in the LMM diagnostic package. The laser tweezers will be used to study the physical effects of microgravity on colloidal crystals. The tweezers are capable of measuring crystal properties, such as yield strength, and are also capable of manipulating crystals by creating defects through the creation of multiple traps. ${ }^{12}$ PHASE-2 and PCS-2 will utilize the tweezers to create an array of optical traps to control a specific colloidal crystal structure and study the micro-rheological properties of the colloid crystal.

Laser tweezers is simply the trapping of a colloidal particle using radiation pressure by focusing a laser beam through a high-numerical aperture lens and striking the particle. Laser tweezers will be implemented using a custom-built system based upon a $1064 \mathrm{~nm} \mathrm{Nd:YAG}$ laser, beam focusing optics, and two acousto-optic deflectors to steer the trap within the field of view of the microscope. The tweezer couples with the microscope through an existing lateral port. A sidelooking dichroic mirror mounted with the fluorescence turret provides the ability to perform normal microscope viewing or confocal microscopy while the laser tweezers are operational.

The laser tweezers have two elements of control. Changing the output laser intensity via commands to the laser controller varies the trapping force. The scanning trap position is controlled with two acoustooptic modulators, or deflectors, which diffract the beam with an acoustic wave in the sample. The frequency of the acoustic wave is modulated via a voltage-controlled oscillator.

Tweezers will also be employed to displace a particle by one or more lattice constants from its equilibrium position. Typical samples are fluorescently dyed PMMA particles, 2.3 microns in diameter, in a nearly index-matching mixture of decalin and tetralin. An image of a trapped array of particles is shown in Figure 9. The tweezers will be scanned through a fixed array of points across the field of view to induce patterns that are either commensurate or incommensurate with the equilibrium configuration of the colloidal crystal. Laser tweezers also will be used to measure the viscosity of the fluid. A particle is trapped and video images taken as it is translated in an oscillatory fashion through the field of view. The velocity just before the particle falls out of the trap is measured from the video record and, along with the known force and particle diameter, used to calculate the sample dynamic viscosity (or crystal shear modulus). 


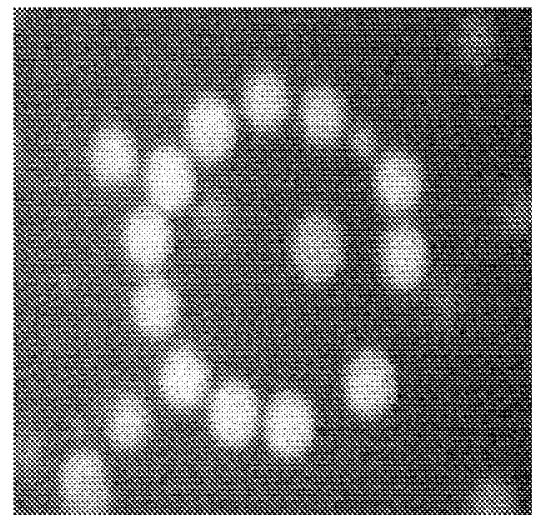

Figure 9 - Example of Multiple Trap

A series of images was taken of the tweezer beam incident on a flat mirror, with a coverglass, at different beam positions within the field of view. The resulting set of images is similar to an "image cube" of the type obtained through confocal imaging. A slice in ' $z$ ' taken through the stack is shown in Figure 10. The contrast has been adjusted, but it clearly shows the intensity gradient of the beam near focus. Also seen in the Figure is the increasing asymmetry of the trap as the position is moved away from the center of the field of view. Figure 10(a) is an x-z slice of the beam in the neutral position, while 10 (b) is a slice taken of the beam when displaced 7 microns and $10(\mathrm{c})$ when displaced 14 microns. The spot position in Figure 10(c) is near the edge of the useable range of motion for the tweezers, as observed during testing. Although it may appear as though the spot size decreases as the tweezer beam moves away from the neutral position, only the intensity is decreasing, thus giving an apparent decrease in spot size.

Measurement of the trap strength required the creation of standard samples to tweeze. The trap strength is a function of particle size, index mismatch between the particle and fluid, trap location due to nonuniform AOD response, axial versus transverse movement, and tweezer misalignment to the microscope. Six slides were created, three containing borosilicate glass spheres of average diameter 2.5 microns, while three others contained glass spheres of diameter 5 microns. The spheres were immersed in calibrated index fluids corresponding to index mismatches at the Sodium D line of $\Delta \mathrm{n}=0.01,0.05$, and 0.1 . The refractive index of the spheres was measured to be 1.56 , as determined by use of index matching fluids. Because the tweezer operates at $1064 \mathrm{~nm}$ rather than the $589 \mathrm{~nm} \mathrm{D}$ line, the actual index mismatches as seen by the tweezer beam were slightly different. For all measurements, an image
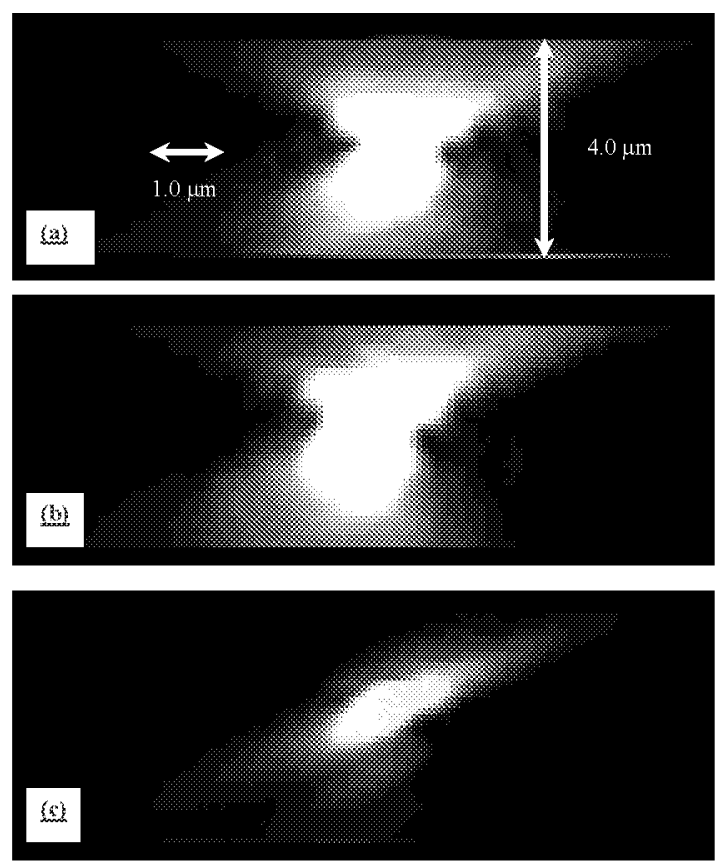

Figure 10 - Laser Tweezer Spot X-Z Profile

(a) neutral position. (b) trap displaced in ' $\mathrm{x}$ ' $7 \mu \mathrm{m}$.

(c) trap displaced in ' $\mathrm{x}$ ' $14 \mu \mathrm{m}$.

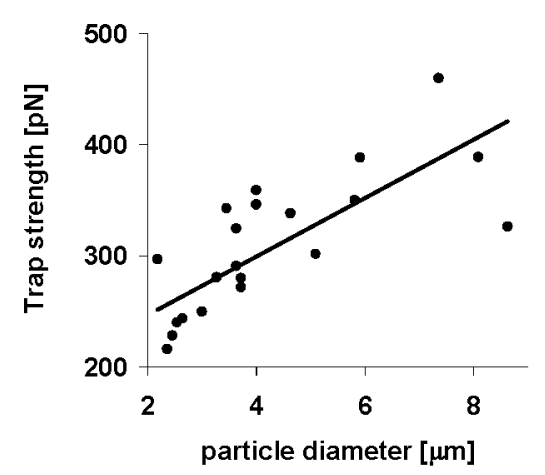

Figure 11 - Laser Tweezer Trap Strength

of the trapped particle was taken for an accurate determination of particle radius.

A summary of test results is shown in Figure 11. It was found during the course of testing that the sample slides with an index mismatch of 0.05 were ideal for use. The higher index mismatched samples were unsuitable due to the lensing effect of the microspheres: the intense field at focus would actually catastrophically burn either the spheres or the fluid. 
The samples at an index mismatch of 0.01 still exhibited trapping, but the mismatch was so small that trapping the particles normally presented difficulties.

\section{$\underline{\text { Confocal }}$}

Confocal microscopy is another research technique planned for implementation within the LMM. Three fluid physics experiments, PHASE-2, PCS-2, and LФCA, scheduled for the LMM will employ the technique to probe the interior structure of complex colloidal systems in order to produce detailed three dimensional models of the unique structures formed in microgravity. Confocal microscopy is generally used to view a fluorescent sample and allows images to consist of only a thin slice $\overline{\bar{q}}_{\bar{q}}$ of a thick sample. These thin slices are then built into a stack of 2-D images, creating a three-dimensional cube. Figure 12 shows the kind of image that can be obtained with a confocal microscope

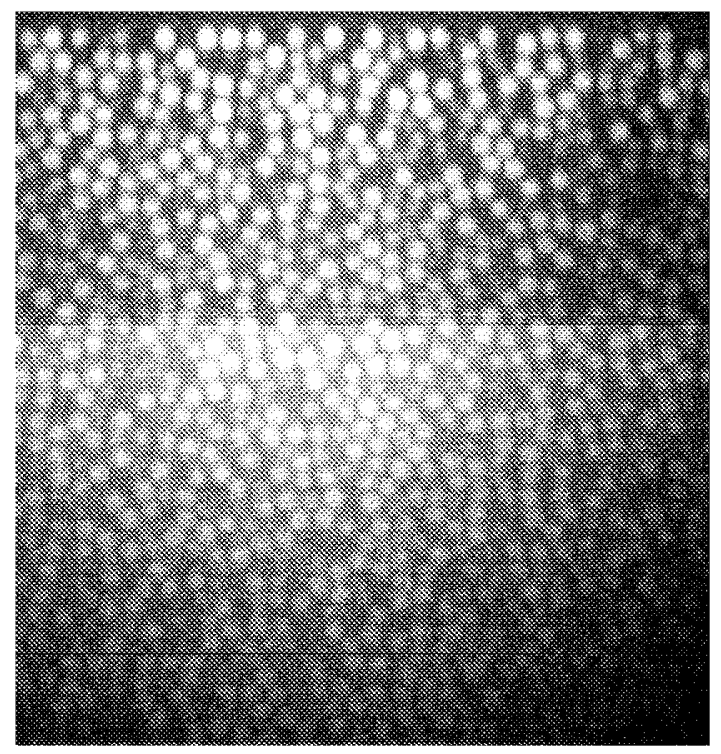

Figure 12 - Two micron diameter PMMA spheres dyed with rhodamine ( $\mathrm{x}-\mathrm{z}$ slice)

Within the LMM, confocal microscopy will be implemented using commercial off-the-shelf subsystems modified to allow non-standard operating configurations. The design involves a FIR provided $532 \mathrm{~nm}$ frequency-doubled Nd:YAG laser, a Yokogawa Model CSU10 Confocal Scanner, a Leica RXA upright microscope, and QImaging Retiga 1300, 12 bit digital CCD camera.

The Yokogawa CSU10 confocal unit used on the LMM is a Nipkow disk-based scanner. The Nipkow disk version of confocal microscopy was chosen for the
LMM due to its inherent stability, speed, and simplicity. It uses a spinning array of matched apertures and lenses to individually map regions of the sample onto the CCD array. The rotational speed of the scanner will allow 30 frames per second of confocal images to the CCD camera. The crystal three-dimensional structure is reconstructed by assembling the slices with an image analysis program, from which colloidal growth, structure, and dynamics can be measured. The confocal module will be attached to the LMM using slide rails, and will access the sample though an auxiliary port on the Leica RXA.

Confocal microscopy generally has advantages over other microscopy techniques such as phase contrast, differential interference contrast, and fluorescence due to the enhanced rejection of out-of-focus light provided by the confocal technique. It results in a much finer resolution in the sectioning dimension (through the sample), allowing better reconstruction of crystal structure and lattice positions, even within somewhat turbid samples. The data is acquired by focusing at the near side of the sample and scanning a two-dimensional image at each focal plane as the focus is translated through to the far side of the sample.

\section{Spectrophotometry}

The spectrophotometer diagnostic capability will measure the photonic properties of crystals grown in the microscope sample cells. Different crystal and alloy structures have various spectral transmission characteristics, which can be analyzed using this technique. The photonic samples will be transilluminated over a range of wavelengths, some of which will transmit through the sample with both a different intensity and at a different scattering angle. The static light scattering distribution will be detected with a high resolution QImaging Retiga 1300 CCD array.

The spectrophotometer monochromatic input will be coupled to the epi-illumination port on the LMM microscope. Spectrophotometry will be implemented using the metal halide lamp in epi-illumination, a monochrometer for visible wavelength discrimination, a translating pinhole in the aperture plane, and the 12 bit digital QImaging Retiga 1300 CCD camera. The monochrometer is used to select narrow-band visible light (10nm bandwidth) from the collimated light in the epi-illumination path and pass it to the pin hole and on to the sample. The translating pinhole is planned to be adjustable in the $\mathrm{X}$ and $\mathrm{Y}$ directions to vary the angle of incidence (up to \pm 65 degrees for normal incidence angle) at the sample. Since this technique is looking at the reflected light coming back from the sample, the detector will be seeing the stop bands of the photonic 
crystals. When the monochrometer is tuned to a wavelength that is in the pass band of the photonic crystal, the light will pass through the crystal and only non-specular reflection will reach the detector.

\section{$\underline{\text { Summary }}$}

The FIR will provide common laboratory diagnostic hardware, in a flexible environment, in order to accommodate a variety of fluid physics experiments. The optics bench will allow for precision mounting of diagnostic hardware. In addition to providing a suite of diagnostic tools for use by the payloads, the FIR's flexible interfaces will allow payloads to utilize experiment unique diagnostic hardware.

The FIR's avionics provides a customizable control and data acquisition system. The FIR avionics can be augmented by payload unique data acquisition and control hardware. For experiment control, the system will allow payload specific software to be uploaded providing the unique control algorithms required to operate the FIR and payload hardware.

The LMM, the first payload on FIR, is a sophisticated, modified commercial research imaging light microscope with laser diagnostics hardware and interfaces to conduct on-orbit colloid, fluid physics, and biological experiments, creating a one-of-a-kind, stateof-the-art microscopic research facility. There are currently five fluid physics experiments planned to be the first to use the facility. The experiment-unique sample cells, procedures, and any specialized measurements are customized within the constraints of the LMM, FIR, and ISS for each Principal Investigator.

The LMM provides imaging techniques of color video microscopy, bright field, dark field, phase contrast, differential interference contrast (DIC), interferometry, confocal microscopy, and spectrophotometry $y_{\tilde{s}}$ all combined in a single configuration. Integrated with the diagnostics is single-trap scanning laser tweezers; to perform sample particle manipulation and patterning. Demonstrations of the LMM preliminary design have been conducted and shown to meet the needs of the Principal Investigators.

\section{$\underline{\text { References }}$}

1. Zurawski, R.L., "The ISS Fluids and Combustion Facility: Microgravity Combustion Science and Fluid Physics Research Capability," AIAA-20014925, Conference \& Exhibit on International Space Station Utilization-2001, October 2001.

2. Wayner Jr., P.C., "The Effect of Interfacial Mass Transport on Flow in Thin Liquid Films," Coll. and Surfaces, Vol. 52, p. 71, 1991.

3. Wayner Jr., P.C., "Effect of Thin Film Heat Transfer on Meniscus Profile and Capillary Pressure," AIAA Journal, Vol. 17, pp. 772-776, 1979.

4. Doherty, M.P., Lant, C.T., Ling, J.S., "The Physics of Hard Spheres Experiment on MSL-1: Required Measurements and Instrument Performance," AIAA-98-0462, 1998.

5. Russel, W.B., Seville, D.A., Schowalter, W.R., "Colloidal Dispersions," Cambridge U. Press, Cambridge, 1995

6. Chaikin, P.M., Lubensky, T.C., "Principles of Condensed Matter Physics," Cambridge U. Press, Cambridge, 1995.

7. Ansari, R.R., Hovenac, E.A., Sankaran, S., Koudelka, J.M., Weitz, D.A., Cipelleti, L., Segre, P.N., "Physics of Colloids in Space Experiment," Space Technology and Applications International Forum-STAIFF-99, 1999.

8. Dinsmore, A.D., Crocker, J.C., Yodh, A.G, "Self Assembly of Colloidal Crystals," Curr Opin Colloid Interface Sci 3, 5-11, 1999.

9. Gast, A.P., "Science Requirements Document Anistropic Colloidal Self Assembly," March 2002.

10. Motil, S.M., Gati, F.G., Snead, J.H., Hill, M.E., Griffin, D.W., "The Fluids Integrated Rack and Light Microscopy Module Integrated Capabilities," World Space Congress, IAC-02-J.5.06, 2002.

11. Eustace, J.G., "CVB Interferometric Liquid Film Metrology Capability Demonstration," LMMTRT-0056, August 2001.

12. Resnick, A.H., "Baseline Performance Light Microscopy Module," LMM-DOC-0021, September 2001. 
\begin{tabular}{|l|l|l|}
\hline 1. AGENCY USE ONLY (Leave blank) & 2. REPORT DATE & 3. REPORT TYPE AND DATES COVERED
\end{tabular}

\begin{tabular}{|l|c|r|}
\hline & January 2003 & Technical Memorandum \\
\hline
\end{tabular}

\begin{tabular}{|l|l|l|}
\hline 4. TITLE AND SUBTITLE & 5. FUNDING NUMBERS
\end{tabular}

The Light Microscopy Module Design and Performance Demonstrations

6. AUTHOR(S)

WBS-22-400-32-30-03

Susan M. Motil, John H. Snead, DeVon W. Griffin, and Edward A. Hovenac

7. PERFORMING ORGANIZATION NAME(S) AND ADDRESS(ES)

National Aeronautics and Space Administration

John H. Glenn Research Center at Lewis Field

Cleveland, Ohio 44135-3191

8. PERFORMING ORGANIZATION

REPORT NUMBER

E-13755

9. SPONSORING/MONITORING AGENCY NAME(S) AND ADDRESS(ES)

10. SPONSORING/MONITORING
AGENCY REPORT NUMBER

National Aeronautics and Space Administration

Washington, DC 20546-0001

NASA TM-2003-212112

AIAA-2003-1303

\section{SUPPLEMENTARY NOTES}

Prepared for the 41st Aerospace Sciences Meeting and Exhibit sponsored by the American Institute of Aeronautics and Astronautics, Reno, Nevada, January 6-9, 2003. Susan M. Motil, John H. Snead, and DeVon W. Griffin, NASA Glenn Research Center; Edward A. Hovenac, Northrop Grumman Information Technology, Brook Park, Ohio 44142.

Responsible person, Susan M. Motil, organization code 6728, 216-433-8589.

12a. DISTRIBUTION/AVAILABILITY STATEMENT 12b. DISTRIBUTION CODE

Unclassified - Unlimited

Subject Category: 74

Distribution: Nonstandard

Available electronically at hto:/ghtrs.grcmasia.gow

This publication is available from the NASA Center for AeroSpace Information, 301-621-0390.

13. ABSTRACT (Maximum 200 words)

The Light Microscopy Module (LMM) is a state-of-the-art space station payload to provide investigations in the fields of fluids, condensed matter physics, and biological sciences. The LMM hardware will reside inside the Fluids Integrated Rack (FIR), a multi-user facility class payload that will provide fundamental services for the LMM and future payloads. LMM and FIR will be launched in 2005 and both will reside in the Destiny module of the International Space Station (ISS). There are five experiments to be performed within the LMM. This paper will provide a description of the initial five experiments: the supporting FIR subsystems; LMM design; capabilities and key features; and a summary of performance demonstrations.

14. SUBJECT TERMS

15. NUMBER OF PAGES

Video microscopy; Confocal microscopy; Spectrophotometry; Laser tweezers

17. SECURITY CLASSIFICATION OF REPORT

Unclassified

NSN 7540-01-280-5500

\begin{tabular}{|c|c|}
\hline $\begin{array}{c}\text { 18. SECURITY CLASSIFICATION } \\
\text { OF THIS PAGE } \\
\text { Unclassified }\end{array}$ & $\begin{array}{c}\text { 19. SECURITY CLASSIFICATION } \\
\text { OF ABSTRACT }\end{array}$ \\
Unclassified
\end{tabular}

Unclassified

Unclassified
16. PRICE CODE

20. LIMITATION OF ABSTRACT 